\title{
The 2016 Dr Falk pharma UK/core awards are open for applications
}

For those committed to furthering research or patient care in gastroenterology and hepatology, this year's awards provide the opportunity to achieve national recognition as well as the financial support to take your career further.

This year's prizes comprise:

- A $£ 1000$ prize for the best essay on gastroenterology research personally undertaken by medical students who were on an intercalated $\mathrm{BSc} / \mathrm{MRes} / \mathrm{MSc} / \mathrm{MPH} / \mathrm{MBPhD}^{*}$ course during the previous academic year (2014/2015)

- Four $£ 1500$ bursaries for medical students taking fulltime science degrees (BSc/MRes/MSc/MPH/MBPhD*) focusing on gastrointestinal or liver-related disease in the current academic year (2015/2016)

- Two $£ 2500$ bursaries to support research for F1/F2 doctors who are undertaking research in an area relevant to gastroenterology

- A $£ 1000$ award for primary and secondary care nurses for initiatives that have advanced patient care.

*PhD students should note that they may apply for a bursary only once during their three-year studentship and that they may apply for the essay prize when their $\mathrm{PhD}$ has been completed.

The application deadline is 17:00 on Friday 4 March 2016. Applications received after this time will not be considered.

Download an application form here: http://www. corecharity.org.uk/research/looking-for-funding

\section{DR FALK PHARMA WINNERS 2015}

\section{Dr Falk-Core Medical Student Essay (1 at $\mathbf{f 1 0 0 0 )}$}

Mario Lepore

Medical Research Council, The Gambia Unit, Fajara, The Gambia

Title of the project: The Diagnostic Accuracy of Hepatitis C virus-DNA Quantification Using Dried Blood Spots in Chronic Hepatitis C virus Carriers in The Gambia

Project Supervisor: Professor Simon Taylor-Robinson

\section{Dr Falk-Core Medical Nurse Recognition Prize (1 at $\mathbf{f 1 0 0 0 )}$} Sarah Alison Thomas

Clinical Nurse Specialist/Nurse Endoscopist, Endoscopy Unit, Royal Gwent Hospital

Developed satellite clinics in primary care to empower patients with ongoing symptoms to self manage symptom relapse, use medication effectively and appropriately where required, understand when to report symptoms to general practitioner (primary care physician) (creating expert patients who can partner with healthcare professionals in the management of their symptoms) and support general practitioner (primary care physician) colleagues in the management of this patient group.

\section{Dr Falk-Core Student Bursaries (4 at $\mathbf{f 1 5 0 0}$ each)}

Zhi-Yang Low

Laboratory of Protein Crystallography, Royal Free Campus, University College London

Title of the project: The predisposition to infection in patients with cirrhosis: rationale for drug design based on novel functional and structural studies of the albumin

Project Supervisor: Dr Alun R Coker

Ashley Rajan Retchless

School of Immunity and Infection, University of Birmingham

Title of the project: Lymphocytes as vehicles for Hepatitis C virus transmission to the liver

Project Supervisor: Dr Zania Stamataki

Rosie Simson

Institute of Cellular Medicine, University of Newcastle

Title of the project: Primary human enteroids: A translational research tool to study therapeutic implications of modulating TGF $\beta$ signalling in inflammatory bowel disease

Project Supervisor: Dr Chris Lamb

Jin Quan Nataniel Tan

Laboratory of Protein Crystallography, Royal Free Campus, University College London

Title of the project: Structural and chemical biology of noroviral proteases-a major therapeutic target for structure-based drug design

Project Supervisor: Professor Jon B Cooper

Dr Falk-Core F1/F2 Bursaries (2 at $£ 2500$ each)

Natalie To

St. James's University Hospital, Leeds

Title of the project: Effect of smoking on the natural history of Crohn's disease: A systematic review and meta-analysis

Project Supervisor: Dr Alexander Ford

Diana Evelyn Yung

Royal Infirmary of Edinburgh

Title of the project: Small-bowel capsule endoscopy in the young anaemic patient

Project Supervisor: Dr Anastasios Koulaouzidis

Correspondence to Alice Kington, Core, 3 St Andrews Place, London NW1 4LB, UK; research@corecharity.org.uk

Accepted 20 August 2015

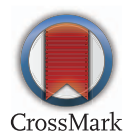

To cite Kington A. Frontline Gastroenterology 2015;6:284. 